\title{
Reverse Shoulder Arthroplasty in Rheumatoid Arthritis: A Systematic Review
}

\author{
Edward C.A. Gee*, Emma K. Hanson and Adnan Saithna
}

Southport and Ormskirk Hospital NHS Trust, Town Lane, Southport, Merseyside, PR8 6PN, UK

\begin{abstract}
Background: Anatomical shoulder replacement for rheumatoid arthritis (RA) is complicated by a high incidence of rotator cuff tears and glenoid erosion. This can lead to poor function and early failure. Reverse shoulder arthroplasty (RSA) has gained popularity as an alternative. This systematic review attempts to further define the role of RSA in RA.

Methods: A systematic review identified seven studies reporting outcomes of RSA in RA patients. Studies were critically appraised, and data on outcomes, complications and technical considerations were extracted and analysed.

Results: One hundred and twenty one shoulders were included (mean follow up 46.9 months). Consistent improvements in the main outcome measures were noted between studies. Ninety five percent of patients described excellent to satisfactory outcomes. The minimum mean forward elevation reported in each study was 115 degrees. Symptomatic glenoid loosening $(1.7 \%)$, deep infection (3.3\%) and revision surgery (5\%) rates were no higher than for a population of mixed aetiologies.

Discussion: Previous concerns regarding high pre- and peri-operative complication and revision rates in RA patients were not shown to be valid by the results of this review. Although associated cuff tears are common and glenoid bone loss can increase the technical complexity of surgery, RSA provides consistent and predictable improvements in key outcome measures and the revision and complication rates do not appear to be higher than reported in a large population of mixed aetiologies.
\end{abstract}

Conclusion: The contemporary literature shows that RSA is a safe, effective and reliable treatment option in RA patients.

Keywords: Outcomes, reverse shoulder arthroplasty, rheumatoid arthritis.

\section{INTRODUCTION}

The glenohumeral joint is frequently affected in rheumatoid arthritis (RA) but the incidence is variably reported. Cuomo et al. found that $90 \%$ of patients with RA had some shoulder impairment. However, this report predates the significant improvements seen in the medical management of RA in the last decade [1]. More recently, Lehtinen et al. reported that $48 \%$ of RA patients developed glenohumeral erosive changes and Rittmeister et al. reported that $75 \%$ of those with seropositive RA developed shoulder symptoms $[2,3]$. Despite this variability, there is no doubt that shoulder involvement is common in RA. In those patients who fail non-operative management, arthroplasty surgery is an option. However, there are several additional concerns when RA patients require shoulder replacement compared to those with glenohumeral osteoarthritis.

The medical management and systemic features of RA create some unique challenges in this population. Diseasemodifying anti-rheumatic drugs are immunosuppressive and are therefore believed to increase the potential risk of prosthetic joint infection. Corticosteroids are also frequently used to control disease progression and long term use affects bone mineral density increasing the risk of fractures and the quality of implant fixation. Furthermore, the non-orthopaedic

*Address correspondence to this author at the Southport and Formby District General Hospital, Town Lane, Southport, Merseyside, PR8 6PN, UK; Tel: (+44) 1704 547471; E-mail: edgee@doctors.org.uk manifestations of the disease increase the risk of systemic peri-operative complications and fitness for anaesthesia.

Historically, anatomical shoulder arthroplasty has played a major role in rheumatoid patients. McCoy et al. found good pain relief in up to $93 \%$ after surgery [4]. However, despite high patient satisfaction and good pain relief, function is unpredictable and often poor, with patients in some series averaging elevation below the horizontal [5-7]. Barrett et al. reported active forward flexion averaged 34 degrees in 140 anatomical shoulder replacements where $50 \%$ of patients had significant cuff pathology [8].

Cuff tears in rheumatoid patients are extremely common $(20 \%-100 \%)[6,9,10]$ and when present, lead to inferior results of joint replacement due to poor pain relief, superior humeral migration, increased shear forces, eccentric wear, instability, early failure and glenoid loosening rates as high as $50 \%[6,7,11-14]$. It is therefore clear that the rotator cuff is an important prognosticator of outcome. Patients with intact cuffs get better pain relief and functional improvement $[6,7,10]$. Although successful outcomes after cuff repair at the time of arthroplasty surgery are reported [15] these results should be interpreted with caution as they may not be applicable to a rheumatoid population in whom progressive upward migration of the humeral head (due to failure of the cuff) has been described as inevitable [10]. Furthermore, the morphology of cuff tears in RA patients more frequently involves Infraspinatus, Subscapularis and Teres Minor and this involvement of a larger part of the cuff may also 
increase the risk of instability and of having a less favourable outcome $[3,16]$.

Moderate to severe erosive changes of the humeral head and glenoid (Larsen grades 3-5) are seen in up to $68 \%$ of rheumatoid patients $[17,18]$. Erosion of the glenoid makes implanting a component more challenging, predisposing to less satisfactory fixation, instability and early implant failure. Previous authors advocated hemi-arthroplasty as the treatment of choice in patients with large glenoid defects, bypassing issues of replacing the eroded socket [19-21]. However functional results were poor and deteriorated with time after the procedure, predominantly due to progressive glenoid wear [21-23]. Some surgeons still favour hemiarthroplasty in rheumatoid patients with an intact coracoacromial arch, accepting that this may later fail [3, 22]. It should be noted that the use of massive allografts (e.g. tendo-achilles), to stabilize shoulders with rotator cuff and glenoid defects has been tried in conjunction with hemiarthroplasty, with dismal results [24].

RSA offers a potential solution for some of these issues. The biomechanical features of this design confer increased stability (particularly against superior escape) and a significantly improved lever arm for deltoid, potentially resulting in improved range and strength of abduction even in the presence of a deficient rotator cuff $[14,25,26]$. Despite these biomechanical improvements, expert authors have previously cautioned against using RSA in RA patients due to concerns about increased peri-operative complications, poor functional outcomes and high revision rates [27, 28]. The aim of this study is to re-visit the evidence for these concerns by combining data from published series and reporting pooled data on outcomes, complications and technical considerations.

\section{METHODS}

\section{Search Strategy}

A PubMed search was performed using the terms "reverse shoulder arthroplasty 'or' reverse shoulder replacement" and "rheumatoid arthritis 'or' inflammatory arthritis" on the $1^{\text {st }}$ of April 2015. Original articles published in English that reported outcomes of RSA in rheumatoid patients were included. Studies were excluded if results were combined with populations containing other aetiologies and the authors were unable to separate out the data.

The PubMed "related citations" tool and Google Scholar were used to search for any further potentially eligible articles. The references of all included articles were also searched for any further relevant articles.

\section{Method of Critical Appraisal}

Included studies were appraised by two independent reviewers (EG, EH) with respect to a validated quality assessment scale for retrospective clinical studies developed by Rangel et al. [29]. The tool allows identification of the most rigorous evidence by characterising 16 baseline criteria that define thorough and accurate reporting of non-controlled studies. If there was any disagreement between the authors in assigning a score to each paper appraised, a third independent reviewer (AS) made the final decision.

\section{Data Extraction}

The main outcomes extracted include: the incidence of complications, the rate of revision, modes of failure and outcome measures relating to function, pain and satisfaction.

PubMed, Medline and Google Scholar databases searched

Reverse shoulder arthroplasty 'or' reverse shoulder replacement, rheumatoid arthritis 'or' inflammatory arthritis

(25 studies identified)

14 studies not relevant and excluded based on abstract

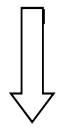

9 citations potentially relevant and retrieved

9 studies excluded:

Patients with other diagnoses/mixed aetiology, data not separated (8)

Case report (1)

Search of references of all citations retrieved

(6 studies identified)

4 excluded

Patients with other diagnoses/mixed aetiology, data not separated (3)

Revision procedures (1)

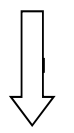

Total of 7 included studies [3,16,30,31,32,33,34]

Fig. (1). Flow of studies. 
Table 1. Adequacy of reporting of studies based on criteria proposed by Rangel et al. [29].

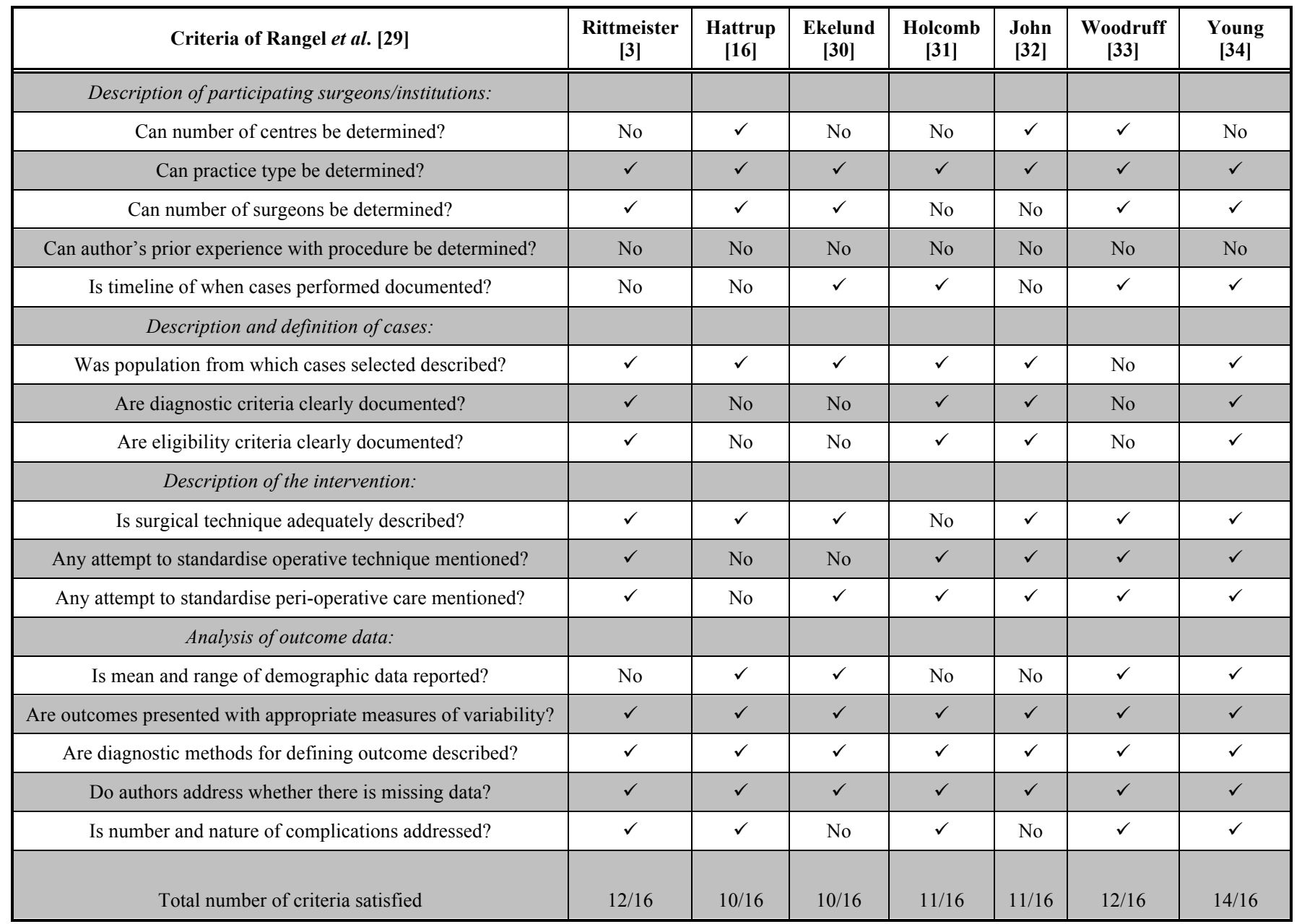

\section{RESULTS}

After application of the search strategy and eligibility criteria seven studies were included for critical appraisal and data extraction [3, 16, 30-34]. The flow of studies is shown in Fig. (1).

The quality assessment of included studies is presented in Table 1. This shows that included studies were of similar quality and that although there were some weaknesses, there were no major limitations in key areas. Data from these studies were collated to produce an overall study population for this review. This is summarised in Table $\mathbf{2}$ and comprises a total of 144 shoulders of which 129 had rotator cuff tears identified on pre-operative imaging. After loss to follow up, 121 reverse arthroplasty shoulders were included with weighted mean follow up of 46.9 months (11.5-143 months). The weighted mean age of patients was 64.6 (range 34-86).

\section{Patient-Based Outcome Measures}

No single outcome measure was universally reported but all authors reported a significant improvement in the main outcome measure used in their studies. Due to the narrow eligibility criteria used in this review, as evidenced by the
Table 2. Summarises basic characteristics of included studies.

\begin{tabular}{|c|c|c|c|c|}
\hline Study & Year & $\begin{array}{c}\text { Level of } \\
\text { Evidence* }\end{array}$ & $\begin{array}{c}\text { No. of } \\
\text { Shoulders }\end{array}$ & $\begin{array}{c}\text { Mean } \\
\text { Follow-Up } \\
\text { (Months) }\end{array}$ \\
\hline \hline Rittmeister [3] & 2001 & IV & 6 & $54.3(48-73)$ \\
\hline Hattrup [16] & 2012 & IV & 19 & $37(24-66)$ \\
\hline Ekelund [30] & 2011 & IV & 27 & $56(18-143)$ \\
\hline Holcomb [31] & 2010 & IV & 21 & $36(24-73)$ \\
\hline John [32] & 2010 & III & 17 & $24.3(11.5-40.9)$ \\
\hline Wooduff [33] & 2003 & IV & 13 & $87(60-110)$ \\
\hline Young [34] & 2011 & IV & 18 & $46(25-84)$ \\
\hline
\end{tabular}

*Oxford Centre for Evidence-Based Medicine 2011 Levels of Evidence.

small number of included patients, it was considered that the included populations were comparable and that some data could be pooled for further analysis. This is evidenced by the fact that the pre- and post- operative scores for the main outcome measures reported were remarkably similar between studies. This data is presented in Table $\mathbf{3}$.

The Constant and Murley Score (CMS) evaluates pain, activities of daily living, mobility and strength and was 
Table 3. Summary of main outcomes data from included studies.

\begin{tabular}{|c|c|c|c|c|c|c|c|c|}
\hline \multirow{2}{*}{ Study } & \multicolumn{3}{|c|}{ CS } & \multicolumn{3}{|c|}{ ASES } & \multirow{2}{*}{$\begin{array}{l}\text { Other Objective } \\
\text { Outcome Measure }\end{array}$} & \multirow{2}{*}{ Patient Satisfaction } \\
\hline & Pre-Op & Post-Op & Mean Difference & Pre-Op & Post-Op & Mean Difference & & \\
\hline Rittmeister [3] & 17 & 63 & 46 & - & - & - & - & Overall: $100 \%, n=6$ \\
\hline Hattrup [16] & - & - & - & 27 & 76 & 49 & $\begin{array}{c}\text { VAPS: } 6.5-1 \\
\text { SST: } 1-8\end{array}$ & - \\
\hline Ekelund [30] & 13 & 52 & 39 & - & - & - & VAPS: 8 - 1 & - \\
\hline Holcomb [31] & - & - & - & 28 & 82 & 54 & $\begin{array}{l}\text { VAPS: } 7-1 \\
\text { SST: } 1-7\end{array}$ & $\begin{array}{l}95 \% \text { (20) Satisfied } \\
5 \% \text { (1) dissatisfied }\end{array}$ \\
\hline John [32] & 19 & 59.5 & 40.5 & - & - & $\begin{array}{l}- \\
-\end{array}$ & $\begin{array}{l}\text { SF-36 MSC: } 108 \% \\
\text { SF-36 PSC: } 77 \%\end{array}$ & Overall: $93 \%, n=14$ \\
\hline Woodruff [33] & - & 58.5 & - & - & - & - & $\begin{array}{c}\text { SF-12 PSC } 38.2 \\
\text { SF-12 MSC } 49.13\end{array}$ & \\
\hline Young [34] & 22.5 & 64.9 & 42.4 & - & - & - & MSSS: $68.6 \%$ & $\begin{array}{c}61 \%(11) \text { very satisfied } \\
33 \% \text { (6) satisfied } \\
5 \% \text { (1) dissapointed }\end{array}$ \\
\hline Weighted mean & & & 40.9 & & & 51.6 & & \\
\hline
\end{tabular}

Pre-op - pre-operative, Post-op - post-operative, CS - Constant-Murley Score, ASES - American Shoulder and Elbow Surgeons outcome measure, VAPS - Visual Analog Pain Scale, SST - Simple Shoulder Test, SF-36 MSC - Short Form 36 Mental Score Component, SF-36 PSC - Short Form 36 Physical Score Component, SF-12 PSC - Short Form 12 Physical Score Component, $S F-12$ MSC - Short Form 12 Mental Score Component, MSSS - Mean subjective shoulder score.

reported by four studies $[3,30,32,34]$. The pooled data from these studies included 68 shoulders and the overall weighted mean improvement in the CMS of 40.9 points was seen from 17.4 to 58.3 at an average follow up of 45.3 months for this group of patients.

It is of note that Young et al. separated the patients according to the presence of a normal or atrophied Teres Minor as seen on MRI scan. The mean CMS improved from 28.0 points to 74.3 points in $8 / 15$ shoulders with a normal Teres Minor muscle and from 20.8 to 54.6 points in $7 / 15$ shoulders with an atrophic Teres Minor muscle. The seven shoulders with a normal Teres Minor muscle preoperatively all had a final CMS of $>70$ points, whereas only two of the eight shoulders with Teres Minor atrophy had a final score in this range [34].

Another two studies [16, 31] reported the American Shoulder and Elbow Surgeons (ASES) outcome measure, which evaluates pain and the level of function or disability. These studies included 40 patients and found a weighted mean improvement of 51.6 points from 27.5 to 79.1 .

\section{Patient Satisfaction}

As well as functional outcome, four studies specifically asked patients to grade overall satisfaction $[3,31,32,34]$. Of the 60 patients in these studies, 95\% $(n=57)$ rated their shoulders as excellent to satisfactory, whereas $5 \%(n=3)$ felt the result of their surgery was unsatisfactory. Specific details are not given regarding patients with an unsatisfactory result so causation cannot be identified. Holcomb et al. do, however, give individual outcome measures and the patient with an unsatisfactory result reported no pain, an improvement in ASES score from 20 to 82 and was able to abduct and forward flex to 90 degrees post-operatively [31].

\section{Range of Motion}

Four of the included studies reported range of movement data pre and post-operatively $[16,30,31,34]$. The results are presented in Table 4. However, it was not possible to combine data due to different methods of measuring the range and the results being presented in different units. Despite that, in all four studies, the mean forward flexion improved from less than $80^{\circ}$ to at least $115^{\circ}$. Abduction was seen to improve in the three studies that reported on it, from less than $70^{\circ}$ to more than $100^{\circ}[16,30,31]$.

\section{Complications}

Overall post-operative complications were seen in $19 \%$ $(n=23)$ of the 121 shoulders. The incidence and type of complication is summarised in Table 5. Two patients had more than one complication. In one, a deep infection occurred after failed acromial osteosynthesis, post osteotomy, utilised in a trans-acromial approach [3] In the other, fractures of the greater tuberosity and the scapular spine were seen in a single patient post-operatively [34].

\section{Glenoid Bone Loss and the Requirement for Bone Grafting}

Of the seven included series, four [16, 30, 31, 34] reported on glenoid bone loss visualised on pre-operative imaging. This was identified in 39 of 85 patients $(45 \%)$, but only a small number required bone grafting (Ekelund et al. $\mathrm{n}=4 / 27$, Holcomb et al. $\mathrm{n}=5 / 21$, Young 8/18).

Ekelund et al. assessed glenoid loss on plain film radiographs for 18 patients and found that eight of the 18 patients assessed had glenoid erosion. Three patients received structural bone grafting and one received cancellous 
Table 4. Mean values for range of movement in operated shoulder. Pre-operative, post-operative and the change in range. Rotation values in Ekelund et al.'s paper are given in Constant Murley Score (CMS) point form.

\begin{tabular}{|c|c|c|c|c|c|c|c|c|c|c|c|c|}
\hline & \multicolumn{3}{|c|}{ Hattrup [16] } & \multicolumn{3}{|c|}{ Ekelund [30] } & \multicolumn{3}{|c|}{ Holcomb [31] } & \multicolumn{3}{|c|}{ Young [34] } \\
\hline & Pre-Op & Post-Op & Change & Pre-Op & Post-Op & Change & Pre-Op & Post-Op & Change & Pre-Op & Post-Op & Change \\
\hline $\mathrm{FF}$ & $68^{\circ}$ & $138^{\circ}$ & $70^{\circ}$ & $33^{\circ}$ & $115^{\circ}$ & $82^{\circ}$ & $52^{\circ}$ & $126^{\circ}$ & $74^{\circ}$ & $78^{\circ}$ & $139^{\circ}$ & $61^{\circ}$ \\
\hline $\mathrm{ABD}$ & $66^{\circ}$ & $134^{\circ}$ & $68^{\circ}$ & $26^{\circ}$ & $103^{\circ}$ & $77^{\circ}$ & $55^{\circ}$ & $116^{\circ}$ & $61^{\circ}$ & - & - & - \\
\hline ER & $23^{\circ}$ & $52^{\circ}$ & $29^{\circ}$ & 0.6 & 5.8 & - & $19^{\circ}$ & $33^{\circ}$ & $14^{\circ}$ & $15^{\circ}$ & $20^{\circ}$ & $5^{\circ}$ \\
\hline IR & - & - & - & 2.1 & 2.9 & - & S1 & L4 & - & sacrum & L3 & - \\
\hline
\end{tabular}

FF - Forward Flexion, ABD - Abduction, ER - External Rotation, IR - Internal Rotation.

graft. One patient also required augmentation of a defect with cement to achieve stability of an inferior screw [30].

Table 5. Summary of Post-operative complications from all papers $[3,16,30-34]$.

\begin{tabular}{|c|c|}
\hline Post-Operative Complication & Overall Incidence \\
\hline \hline Infection and septic loosening & $3.3 \%, \mathrm{n}=4$ \\
\hline Scapular Spine fracture & $3.3 \%, \mathrm{n}=4$ \\
\hline Glenoid fracture & $3.3 \%, \mathrm{n}=4$ \\
\hline Failed Acromion Osteosynthesis & $2.5 \%, \mathrm{n}=3$ \\
\hline Symptomatic aseptic glenoid loosening & $1.7 \%, \mathrm{n}=2$ \\
\hline Greater tuberosity fracture & $1.7 \%, \mathrm{n}=2$ \\
\hline Acromial fracture & $1.7 \%, \mathrm{n}=2$ \\
\hline Nerve Palsy & $1.7 \%, \mathrm{n}=2$ \\
\hline Dislocation & $0.8 \%, \mathrm{n}=1$ \\
\hline Coracoid fracture & $0.8 \%, \mathrm{n}=1$ \\
\hline Symptomatic aseptic humeral loosening & $0 \%, \mathrm{n}=0$ \\
\hline Total & 25 complications \\
& $(23$ shoulders $=19 \%)$ \\
\hline
\end{tabular}

Holcomb et al. measured available glenoid bone stock (CT/MRI) from the most medial portion of the glenoid to the medial base of the coracoid process, with a mean of $24.2 \mathrm{~mm}$ (range 11.2-30.5) remaining. The need for grafting wasn't based on these values but on intra-operative visual inspection, if less than $80 \%$ of the baseplate had osseous contact, bone grafting (humeral head autograft) was used. Stable fixation of the baseplate was achieved in each case with no cases of non-union of the bone graft [31].

Young et al. also assessed glenoid loss using MRI or CT imaging according to Levigne's classification. Fourteen patients $(78 \%)$ had glenoid erosion. Eight of these were treated intra-operatively with bone grafting, four using cancellous bone autograft and four requiring structural autografting, from the resected humeral head [34] At final follow up, all patients that had received bone grafts had a well fixed glenoid component on plain film radiography.

Hattrup et al. took a different approach to managing glenoid erosion and did not use structural bone grafts. Instead, the inferior margin of the glenoid baseplate was aligned with the inferior margin of the glenoid and reaming was directed to create neutral orientation and over $50 \%$ bony contact with the baseplate. No attempt was made to create an inferior tilt for the glenosphere to avoid exacerbating superior bone loss. Cancellous autograft from the humeral head was used to fill residual glenoid defects [16].

\section{Scapula Notching}

Notching is a well-recognised consequence of RSA, but the clinical significance is unclear [35, 36] Only four of the included studies reported scapula notching, at a rate of $42 \%(n=34 / 81)[16,30,32,34]$.

The majority of these (21) were grade 1-2 according to the Nerot-Sirveaux classification (appendix) [37]. Thirteen patients had notching above the inferior glenoid component screw. All notching was asymptomatic with no glenoid loosening or fracture being reported as associated with notching within the study periods.

\section{Infection}

The overall rate of deep infection in the combined series was $3.3 \%(n=4)$ and all four required further surgery [3, 30, 31]. This is comparable to infection rates for this operation for other aetiologies. One patient from the series reported by Ekelund et al. underwent a successful two-stage revision after developing deep infection within the first year. Causative organisms were not reported. The ConstantMurley score at latest follow up was 48 points [30].

In Holcomb's series, one patient developed deep infection (Pseudomonas aeruginosa) at six years postoperation, requiring removal of the prostheses and a hemiarthroplasty cement spacer. Another patient with early infection (Methicillin-sensitive Staph. Aureus) diagnosed at seven weeks required washout, debridement and exchange of polyethylene liner along with six weeks of antimicrobial therapy. This patient was infection free at final follow up (two years) and rated the outcome as 'excellent' [31].

In the Rittmeister et al. series, one patient developed sepsis within the joint after re-operation for non-union of acromial osteosynthesis (post osteotomy). This patient required implant removal [3]. Only Ekelund et al. reported their antibiotic prophylaxis regimens (Flucloxacillin, or Clindamycin if penicillin allergic) [30]. Holcomb et al. advocated the routine inclusion of Tobramycin into bone cement [31]. No clues as to causation of infection can be drawn from the data, nor do the authors postulate causation. 


\section{Loosening}

There were no cases of symptomatic humeral loosening in the combined series. Twenty cases of radiographic humeral loosening were reported but none of these had symptoms at final review $[16,33]$. Woodruff et al. found that $100 \%$ of their cases had radiolucencies surrounding the uncemented, hydroxyapatite coated humeral components (Delta III) at a mean follow up of 87 months. These may be attributed to stress shielding rather than true loosening and osteolysis.

There were seven (overall incidence 5.7\%) cases of glenoid loosening reported. Once again the majority (five patients) were found in the series reported by Woodruff et al. and none of these were symptomatic. One of these occurred in a patient that sustained intra-operative fracture of the glenoid [33]. Of the other two, one still rated the shoulder as good [31], the other resulted in breaking of the inferior screw and tilting of the component which required revision [3].

\section{Fracture}

Thirteen (10.7\%) fractures occurred associated with RSA in this group of patients. Four patients sustained an intraoperative glenoid fracture during glenoid reaming. Holcomb et al. fixed the two intra-operative fractures occurring in their series with cannulated lag-screws, allowing subsequent stable implantation of a glenoid component and excellent post-operative results (ASES 80, forward flexion 125 degrees) [31]. Ekelund et al. [30] and Woodruff et al. [33] both had one intra-operative fracture, neither paper specifically discusses intra-operative fixation or alteration to post-operative management. However, Woodruff, et al. reported that this patient had a very deficient glenoid at the time of surgery and found loosening of the glenoid component and a Constant score of 51 at final follow up.

Five fractures were seen in Young's series, two sustaining post-operative acromial fractures, one resulted in inferior tilt of the fragment; the patient rated her outcome as satisfactory, and reported no pain and had active abduction of $140^{\circ}$ at the latest follow-up. The other was in the acromial spine; this healed with conservative management but unfortunately led to reduced mobility and the patient reported pain for one year. Two patients sustained avulsion fractures of the greater tuberosity that were thought to have occurred intra-operatively but were not identified at the time. One of these occurred in the patient with an acromial spine fracture and contributed to the poor outcome. The other healed and reported good outcomes. Another patient sustained a coracoid avulsion that was repaired at the time with sutures and healed [34].

In other studies, three more scapular spine fractures occurred after falls; one healed giving excellent results (forward flexion 160 degrees, ASES 83), one was discovered after bone scan for progressive pain, another did not heal and gave a poor outcome with pain score of 10 and ASES of 7 (not revised at final follow up). One patient sustained a stress fracture of the acromion at eight months and although this didn't heal, the patient could forward flex to 100 degrees and the ASES was $60[16,30,31]$.

\section{Rates of Revision Surgery and Modes of Failure}

Rates of revision surgery in the included studies varied from $0-38 \%$ at latest follow up. The overall rate of revision was $7 \%(n=9)$ at a mean follow up of 46.9 months. However, if the series of six patients reported by Rittmeister et al. [3] is excluded, on the basis that it is not contemporary and represents a significant outlier compared to recent studies, then the mean rate of revision is only $5 \%(n=6 / 115)$ in this population at a mean follow up of 46.5 months. Three cases required revision for septic loosening, 1 for instability, 1 for glenoid fracture and 1 due to breakage of the central screw. The three re-operations (50\%) described by Rittmeister et al. were all related to failed osteosynthesis of the acromion after osteotomy in the trans-acromial approach, used for better exposure, thus adding further weight to the decision to exclude that study in the analysis of revision surgery.

A systematic review by Zumstein et al. of 782 patients receiving RSA for all aetiologies, revealed an overall revision rate of $10.1 \%$, which is higher than the rate we found in RA patients [38].

\section{DISCUSSION}

RSA is increasingly advocated as an appropriate option for primary arthroplasty in end-stage RA. Of only seven available series specifically focusing on this indication, five were published in the last four years demonstrating a recent increase in popularity for this treatment option.

This review reports outcomes collated from the seven available studies. The most commonly used outcome measure was the Constant-Murley Score (CMS). This includes functional assessment, range of motion and strength of active abduction as well as the pain score [39, 40]. All studies that reported this outcome measure showed a remarkably consistent improvement at a mean of 41.62 (range 39 to 46) points. This is comparable with studies looking at RSA in cuff tears (mean improvement of 43) [19, $41,42]$ or cuff arthropathy (mean improvement of 41.33) [43-45] and suggests that outcome from RSA in RA is predictable.

The American Shoulder and Elbow Surgeons (ASES) outcome measure concentrates more on subjective functional limitations of the shoulder and pain associated with it. Studies included in our review showed an overall mean improvement in ASES scores of 51.6 points (from 27.5 to 79.1), which, although not directly comparable, is greater than the improvement reported in RSA performed for cuff arthropathy (40.75) [46-49].

John et al. reported results from the Short-Form 36 (SF36). Although there was some improvement in physical function, a great improvement was seen in the mental and emotional components, above that of a normative population. The authors postulated this might be due to lower expectations of function in RA patients and an increase in satisfaction once pain had been relieved, despite still having a functional impairment [32]. This theory can also be used to explain high satisfaction rates seen in this lower demand and lower expectation cohort burdened with chronic functional impairment and pain. 
Range of movement was seen to improve in all mean values in the four studies in which they were reported [16, $30,31,34]$. It was not possible to combine data for analysis but there are some key points to note. Firstly, as stated in the introduction forward elevation in RA patients after TSA or hemiarthroplasty can be extremely poor [5-8, 21-23]. Khan compared osteoarthritic patients to RA patients undergoing anatomical total shoulder arthroplasty and found postoperative forward elevation averaging $130^{\circ}$ and $85^{\circ}$ respectively [6] Table 4 demonstrates that the authors of the studies reporting on range of motion included in this review all recorded a minimum mean forward elevation of 115 degrees. Although a comparative study is required to demonstrate a significant difference in ROM this data certainly reinforces the empirical observation of many surgeons that RSA allows greater potential for forward elevation than anatomical replacement in an RA population due to the high incidence of associated cuff tears.

Furthermore, although Young et al. found little improvement in external rotation with the arm in an adducted position, with mean external rotation improving from $15^{\circ}$ to $20^{\circ}$ of rotation, they also tested 'functional external rotation', with the arm at $90^{\circ}$ abduction. They found a greater improvement from $17^{\circ}$ pre-operatively to $46^{\circ}$ functional external rotation post-operatively. The authors attributed this difference to the presence of a functioning Teres Minor muscle facilitating external rotation with the arm in this position. This also accounted for the difference in CMS scores reported in this paper [34].

Shoulder arthroplasty complication rates have declined in recent years with a lower need for re-operation. It is stated by some authors that there is no discernable link between early or late complications and initial aetiology, with comparable complication rates occurring in RSA regardless of whether the patient has RA [50]. In order to ascertain whether the perceived increase in risk of complication, when performing RSA in RA patients, does exist, comparisons can be made to RSAs performed for mixed aetiologies. A systematic review by Zumstein et al. looked at the results of 782 RSAs in 761 patients performed for multiple aetiologies, including cuff tears, fracture, tumour, revision surgery and RA [38].

The overall complication rate in this series of patients was 19\% (23/121 shoulders). Wall et al. reported a similar complication rate of $19 \%$ in a series of 191 reverse arthroplasties [51] and Frankle et al. found a complication rate of $17 \%$ [52]. Zumstein et al. actually found a higher rate of complication in their systematic review of 782 RSAs for all aetiologies. They separated 'problems' and 'complications', based on whether they thought the final outcome was likely (complication) or unlikely (problem) to be affected. They reported an overall 'problem' rate of $44 \%$ and a 'complication' rate of $24 \%$ in a mixed population [38].

One of the main concerns when pre-operatively planning shoulder arthroplasty in RA patients is glenoid bone loss, and this was apparent in $45 \%$ of patients in this cohort. Less than $20 \%$ of those with bone loss (9\% overall) required grafting. By final follow up, all these patients had a well fixed and stable glenoid component with no signs of nonunion or early loosening, suggesting that this is a useful option for managing deficient glenoid bone stock.
The rate of scapula notching that occurred in the combined series was 34\%. Prior clinical studies have shown scapular notching rates ranging from $0 \%$ to $96 \%$ in RSA [53]. Notching is usually apparent within the first few postoperative months and very few shoulders that have avoided notching by 12 months demonstrate notching later [28, 42, 54]. This suggests that the length of follow up in the combined series was of sufficient duration to appropriately capture the incidence of scapula notching and that its incidence was no greater than that seen when RSA was performed for all aetiologies (35\%) [38]. No direct links were made between notching and fracture, loosening or revision in any of the studies included in this review.

Infection is a devastating complication in arthroplasty. RA patients are potentially immunocompromised and so higher infection rates are anticipated. The overall infection rate in this combined series was $3.3 \%$, which is not dissimilar to a population of mixed aetiologies undergoing the same operation. In previous papers concerning a normal population undergoing primary RSA, infection rate is stated as between $1 \%$ and $5 \%[36,55]$, and Zumstein et al.'s review of 782 RSAs found a deep infection rate of $3.8 \%$ [38]. The infecting organisms described in the patient cohort included in this review were typical, including Pseudomonas acnes, Pseudomonas aeruginosa, Staphylococcus Aureus, and coagulase-negative Staphylococcus [31, 56]. However, rare cases of prosthetic joint infection in RSA implants with Candida Albicans have been reported in RA patients. It is hypothesised that this is almost certainly due to immunosuppression caused by disease controlling drugs such as anti-tumour necrosis factor therapy (e.g. Etanercept) and should also therefore be considered as a potential infecting micro-organism if an RA patient presents with a prosthetic joint infection [57].

Osteopenia and osteoporosis associated with glucocorticoid medication, disuse and the disease process itself, raise concerns of fracture in RA patients. Thirteen fractures were seen in twelve patients within the cohort included in this review, giving a fracture risk of $10.7 \%$ overall. Seven occurred intra-operatively and six occurred post-operatively. Seven of these fractures healed and in general these reported good outcomes, apart from the patient with an acromial spine and a greater tuberosity fracture who had an unsatisfactory outcome. Five fractures did not heal and four of these reported poor outcomes. The healing and outcome of one of the fractures was not reported. Zumstein et al. reported 46 fractures in 782 RSAs in a mixed population, giving an overall fracture risk of 5.9\%, which is lower than seen in the purely RA cohort of this review [38]. As the rate of fracture varied greatly between the studies in this review from $4 \%$ [30] to $28 \%$ [34], it may be that specific aspects of the intra-operative technique or the prosthesis used increased the risk of fracture in certain studies however this cannot be ascertained from the data provided.

There were no cases of symptomatic humeral loosening and only two cases of symptomatic glenoid loosening, one of which required revision in the combined series. Depending on the criteria used for radiographic loosening, extremely high rates of up to $100 \%$ were reported and Woodruff et al. attributed this to stress shielding surrounding uncemented components rather than true osteolysis [33]. Zumstein et al. 
found radiographic loosening in 27 (out of 782) RSAs giving an overall rate of $3.5 \%$ in a mixed aetiology population. Loosening rates varied greatly depending on which prosthesis was used and the fixation type. The authors found loosening rates were twice as high in the Encore system compared to the Grammont system [38].

A final concern with rheumatoid patients is the existence of multi-system involvement and a perceived increased risk of peri-operative systemic complications. None of the included studies reported systemic peri-operative complications. Hambright et al. reported a comparison of peri-operative complications in RA $v s$ non RA patients undergoing shoulder arthroplasty and found this to be less than $1 \%$ in both groups, with no significant difference between them [58].

Due to a limited number of studies providing data on this subject and a variation of follow up, we are unable to report cumulative survivorship. However, the mean rate of revision from the contemporaneous combined series (excluding Rittmeister et al. [3] as previously discussed) was 5\% at a mean follow up of 46.9 months. Although not directly comparable, when one is considering the role of RSA in RA patients it is important to note that the revision rate given for RSA in a mixed population was $10.1 \%$ suggesting that the risk for RA patients is not excessively high [38]. Other studies have also noted that revision rates for anatomical shoulder arthroplasty in RA patients are lower than those published for non-RA patients, possibly due to a lower demand in this cohort or to statistical variance secondary to small sample sizes [21, 59-61].

\section{CONCLUSION}

The contemporary literature does not show a higher rate of complications for RSA in rheumatoid patients either in the peri- or post-operative period when compared to RSA in a large population of patients with mixed aetiologies [38]. Preconceptions of increased rates of infection, fracture, notching, loosening and failure were not realised in this review when compared to other aetiologies.

The data suggests it is a safe procedure that allows predictable improvements in the ROM, CMS and ASES scores as well as high patient satisfaction. Longer-term data, larger numbers and prospective, controlled trials may be beneficial in substantiating this and to better estimate longterm survivorship. This review concludes that RSA is a valuable treatment option for end stage arthritis in rheumatoid patients.

\section{CONFLICT OF INTEREST}

The authors confirm that this article content has no conflict of interest.

\section{ACKNOWLEDGEMENTS}

Declared none.

\section{REFERENCES}

[1] Cuomo F, Greller MJ, Zuckerman JD. The rheumatoid shoulder. Rheum Dis Clin North Am 1998; 24: 67-82.

[2] Lehtinen JT, Kaarela K, Belt EA, Kautiainen HJ, Kauppi MJ, Lehto MU. Incidence of glenohumeral joint involvement in seropositive rheumatoid arthritis. A 15 year endpoint study. J Rheumatol 2000; 27: 347-50.

[3] Rittmeister M, Kerschbaumer F. Grammont reverse total shoulder arthroplasty in patients with rheumatoid arthritis and nonreconstructible rotator cuff lesions. J Shoulder Elbow Surg 2001; 10(1): 17-22.

[4] McCoy S, Warren RF, Bade HA, Ranawat CS, Inglis AE. Total shoulder arthroplasty in Rheumatoid Arthritis. J Arthroplasty 1989; 4: 105-13.

[5] Jolles BM, Grosso P, Bogoch ER. Shoulder arthroplasty in patients with juvenile rheumatoid arthritis. J Arthroplasty 2007; 22: 876-83.

[6] Khan A, Bunker TD, Kitson JB. Clinical and radiological followup of the Aequalis third-generation cemented total shoulder replacement. A minimum ten-year study. J Bone Joint Surg Br 2009;91:1594-1600. J Bone Joint Surg Br 2009; 91(12): 1594-600.

[7] Sperling JW, Cofield RH, Schleck CD, Harmsen WS. Total shoulder arthroplasty versus hemiarthroplasty for rheumatoid arthritis of the shoulder: results of 303 consecutive cases. J Shoulder Elbow Surg 2007; 16(6): 683-90.

[8] Barrett WP, Thornhill TS, Thomas WH, Gerbhart EM, Sledge CB. Non-constrained total shoulder arthroplasty in patients with polyarticular rheumatoid arthritis. J Arthroplasty 1989; 4: 91-6.

[9] Ennevaara K. Painful shoulder joint in rheumatoid arthritis: a clinical and radiographic study of 299 cases with special reference to arthrography of the glenohumeral joint. Acta Rheumatol Scand 1967: (Suppl 11): 111-6.

[10] Lehtinen JT, Belt EA, Lybäck CO, et al. Subacromial space in the rheumatoid shoulder: a radiographic 15-year follow-up study of 148 shoulders. J Shoulder Elbow Surg 2000; 9: 183-7.

[11] Cofield RH. Total shoulder arthroplasty with the Neer prosthesis. J Bone Joint Surg 1984; 66: 899-906.

[12] Franklin JL, Barrett WP, Jackins SE, Matsen FA $3^{\text {rd }}$. Glenoid loosening in total shoulder arthroplasty: association with rotator cuff deficiency. J Arthroplasty 1977; 3(1): 39-46.

[13] Khan WS, Longo UG, Ahrens PM, Denaro V, Maffulli N. A systematic review of the reverse shoulder replacement in rotator cuff arthropathy, rotator cuff tears, and rheumatoid arthritis. Sports Med Arthrosc 2011; 19(4): 366-79.

[14] Smithers CJ, Young AA, Walch G. Reverse shoulder arthroplasty. Curr Rev Musculoskelet Med. 2011; 4(4): 183-90.

[15] Rozing PM, Brand R. Rotator cuff repair during shoulder arthroplasty in rheumatoid arthritis. J Arthroplasty 1998; 13: 311-9.

[16] Hattrup SJ, Sanchez-Sotelo J, Sperling JW, Cofield RH. Reverse shoulder replacement for patients with inflammatory arthritis. J Hand Surg 2012; 37(9): 1888-94.

[17] Larsen A, Dale K, Eek M. Radiographic evaluation of rheumatoid arthritis and related conditions by standard reference films. Acta Radiol Diagn (Stockh) 1977; 18(4): 481-91.

[18] Tanaka H, Sugamoto K, Sahara W, et al. The mode of destruction in shoulders with rheumatoid arthritis based on radiographic findings. J Shoulder Elbow Surg 2007; 16(6): 539-43.

[19] Grassi FA, Murena L, Valli F, Alberio R. Six-year experience with Delta III reverse shoulder prosthesis. J Orthop Surg (Hong Kong) 2009; 17(2): 151-6.

[20] Sojbjerg JO, Frich LH, Johannsen HV, Sneppen O. Late results of total shoulder replacement in patients with rheumatoid arthritis. Clin Orthop Relat Res 1999; 366: 39-45.

[21] Van de Sande MA, Brand R, Rozing PM. Indications, complications, and results of shoulder arthroplasty. Scand J Rheumatol 2006; 35(6): 426-34.

[22] Boyer P, Huten D, Alnot JY. Bipolar shoulder prosthesis for rheumatoid arthritis with irreparable rotator cuff tear: results after 5 years' follow up [Article in French]. Rev Chir Orthop Reparatrice Appar Mot 2006; 92(6): 556-66.

[23] Gregory T, Hansen U, Emery RJ, Augereau B, Amis AA. Development in shoulder arthroplasty. Proc Inst Mech Eng H 2007; 221(1): 87-96. 
[24] Norris TR, Kelly JDI, Humphrey CS. Management of glenoid bone defects in revision shoulder arthroplasty: a new application of the reverse total shoulder prosthesis. Tech Shoulder Elbow Surg 2007; 8: 37-46.

[25] Grammont PM, Baulot E. Delta shoulder prosthesis: biomechanical principles. [Personal communication] 1991.

[26] Grammont PM, Baulot E. Delta shoulder prosthesis for rotator cuff rupture. Orthopedics 1993; 16: 65-8.

[27] Gerber C, Pennington SD, Nyffeler RW. Reverse total shoulder arthroplasty. J Am Acad Orthop Surg 2009; 17(5): 284-95.

[28] Guery J, Favard L, Sirveaux F, Oudet D, Mole D, Walch G. Reverse total shoulder arthroplasty. Survivorship analysis of eighty replacements followed for five to ten years. J Bone Joint Surg Am 2006; 88(8): 1742-7.

[29] Rangel S, Kelsey J, Henry MCW, Moss RL. Critical analysis of clinical research reporting in pediatric surgery: Justifying the need for a new standard. J Pediatr Surg 2003; 38(12): 1739-43.

[30] Ekelund A, Nyberg R. Can Reverse Shoulder Arthroplasty be Used with Few Complications in Rheumatoid Arthritis? Clin Orthop Relat Res 2011; 469(9): 2483-8.

[31] Holcomb JO, Hebert DJ, Mighell MA, et al. Reverse shoulder arthroplasty in patients with rheumatoid arthritis. J Shoulder Elbow Surg 2010; 19(7): 1076-84.

[32] John M, Pap G, Angst F, et al. Short-term results after reversed shoulder arthroplasty (Delta III) in patients with rheumatoid arthritis and irreparable rotator cuff tear. Int Orthop 2010; 34(1): 71-7.

[33] Woodruff MJ, Cohen AP, Bradley JG. Arthroplasty of the shoulder in rheumatoid arthritis with rotator cuff dysfunction. Int Orthop 2003; 27(1): 7-10.

[34] Young A, Smith M, Bacle G, Moraga C, Walch G. Early results of reverse shoulder arthroplasty in patients with rheumatoid arthritis. J Bone Joint Surg Am 2011; 93: 1915-23.

[35] Sirveaux F, Favard L, Oudet, Huquet D, Walch G, Molé D. Grammont inverted total shoulder arthroplasty in the treatment of glenohumeral osteoarthritis with massive rupture of the cuff. J Bone Joint Surg Br 2004; 86: 388-95.

[36] Guery J, Favard L, Sirveaux F, Oudet D, Molé D, Walch G. Reverse total shoulder arthroplasty. Survivorship analysis of eighty replacements followed for five to ten years. J Bone Joint Surg Am 2006; 88: 1742-7.

[37] Sirveaux F. La prothèse de Grammont dans le traitement des arthropathies de l'épaule à coiffe détruite. A propos d'une série multicentrique de 42 cas. Faculté de médecine de Nancy, thèse de l'université de Nancy I 1997; 245.

[38] Zumstein MA, Pinedo M, Old J, Boileau P. Problems, complications, reoperations, and revisions in reverse total shoulder arthroplasty: A systematic review. J Shoulder Elbow Surg 2011; 20: 146-57.

[39] Boehm D, Wollmerstedt N, Doesch M, Handwerker M, Mehling E, Gohlke F. Development of a questionnaire based on the ConstantMurley-Score for self-evaluation of shoulder function by patients. [Article in German]. Unfallchirurg 2004; 107(5): 397-402.

[40] Conboy VB, Morris RW, Kiss J, Carr AJ. An evaluation of the Constant-Murley shoulder assessment. J Bone Joint Surg Br 1996; 78(2): 229-32.

[41] Boileau P, Gonzalez JF, Chuinard C, Bicknell R, Walch G. Reverse total shoulder arthroplasty after failed rotator cuff surgery. J Shoulder Elbow Surg 2009;18(4): 600-6.

[42] Sirveaux F, Favard L, Oudet D, Huquet D, Walch G, Molé D. Grammont inverted total shoulder arthroplasty in the treatment of glenohumeral osteoarthritis with massive rupture of the cuff Results of a Multicentre Study of 80 Shoulders. J Bone Joint Surg Br 2004; 86(3): 388-95.

[43] Boileau P, Watkinson D, Hatzidakis AM, Hovorka I. Neer award 2005: the Grammont reverse shoulder prosthesis: results in cuff tear arthritis, fracture sequelae, and revision arthroplasty. J Shoulder Elbow Surg 2006; 15: 527-40.

[44] Neyton L, Boileau P, Nové-Josserand L, Edwards TB, Walch G. Glenoid bone grafting with a reverse design prosthesis. J Shoulder Elbow Surg 2007; 16(Suppl 3): 71-8.

[45] Sayana MK, Kakarala G, Bandi S, Wynn-Jones C. Medium term results of reverse total shoulder replacement in patients with rotator cuff arthropathy. Ir J Med Sci 2009; 178(2): 147-50.

[46] Cuff D, Pupello D, Virani N, Levy J, Frankle M. Reverse shoulder arthroplasty for the treatment of rotator cuff deficiency. J Bone Joint Surg Am 2008; 90: 1244-51.

[47] Klein SM, Dunning P, Mulieri P, Pupello D, Downes K, Frankle MA. Effects of acquired glenoid bone defects on surgical technique and clinical outcomes on reverse shoulder arthroplasty. J Bone Joint Surg Am 2010; 92: 1144-54.

[48] Mullieri P, Dunning P, Klein S, Pupello D, Frankle M. Reverse shoulder arthroplasty for the treatment of irreparable rotator cuff tear without glenohumeral arthritis. J Bone Joint Surg Am 2010; 92: 2544-56.

[49] Werner CM, Steinmann PA, Gilbart M, Gerber C. Treatment of painful pseudoparesis due to irreparable rotator cuff dysfunction with the Delta III reverse-ball-and-socket total shoulder prosthesis. J Bone Joint Surg Am 2005; 87: 1476-86.

[50] Chin PY, Sperling JW, Cofield RH, Schleck C. Complications of total shoulder arthroplasty: are they fewer or different? J Shoulder Elbow Surg 2006; 15: 19-22.

[51] Wall B, Nové-Josserand L, O’Connor LP, Edwards TB, Walch G. Reverse total shoulder arthroplasty: a review of results according to etiology. J Bone Joint Surg Am 2007; 89: 1476-85.

[52] Frankle M, Siegal S, Pupello D, Saleem A, Mighell M, Vasey M. The reverse shoulder prosthesis for glenohumeral arthritis associated with severe rotator cuff deficiency. A minimum twoyear follow-up study of sixty patients. J Bone Joint Surg Am 2005; 87: 1697-705.

[53] Kempton LB, Balasubramaniam M, Ankerson E, Wiater JM. A radiographic analysis of the effects of glenosphere position on scapular notching following reverse total shoulder arthroplasty. J Shoulder Elbow Surg 2011; 20: 968-74.

[54] Nyffeler RW, Werner CM, Simmen BR, Gerber C. Analysis of a retrieved Delta III total shoulder prosthesis. J Bone Joint Surg Br 2004; 86: 1187-91.

[55] Trappey GJ, O'Connor DP, Bradley Edwards T. What are the instability and infection rates after reverse shoulder arthroplasty? Clin Orthop Relat Res 2011; 469: 2505-11.

[56] Kelly JD II, Zhao JX, Rhett Hobgood E, Norris TR. Clinical results of revision shoulder arthroplasty using the reverse prosthesis. J Shoulder Elbow Surg 2012; 21: 1516-20.

[57] Radha S, Siddiqui BM, Senevirathna S, Harrison JW. Candida albicans infection of a reverse-polarity shoulder replacement in a patient with rheumatoid arthritis treated with etanercept. J Clin Rheumatol 2012; 18(6): 323.

[58] Hambright D, Henderson RA, Cook C, Worrell T, Moorman CT, Bolognesi MP. A comparison of perioperative outcomes in patients with and without rheumatoid arthritis after receiving a total shoulder replacement arthroplasty. J Shoulder Elbow Surg 2011; 20(1): 77-85.

[59] Brenner BC, Ferlic DC, Clayton ML, Dennis DA. Survivorship of unconstrained total shoulder arthroplasty. J Bone Joint Surg Am 1989; 71: 1289-96.

[60] Neer CS II, Kirby RM. Revision of humeral head and total shoulder arthroplasties. Clin Orthop Relat Res 1982; 189-95.

[61] Wirth MA, Seltzer DG, Senes HR, Pannone A, Lee AJ, Rockwood CA. An analysis of failed humeral head and total shoulder arthroplasty [abstract]. J Shoulder Elbow Surg 1995; 4(Suppl 1): S13. 